\title{
MANEJO DA SÍNDROME DA VEIA CAVA SUPERIOR
}

\section{ARTIGO DE REVISÃO}

ALMEIDA, Mariana Ferreira ${ }^{1}$, SOUZA, Ana Paula Barbosa de ${ }^{2}$, ALMEIDA, Bárbara Rezende de ${ }^{3}$, CHOAIRY TERCEIRO, Carim Miguel ${ }^{4}$, GUEDES, Gabriella Thalya ${ }^{5}$, BORGES, Italo Ganam ${ }^{6}$, CHERN, Lucas ${ }^{7}$, CARNEIRO, Luis Eduardo Lima ${ }^{8}$, NETTO, Maria Eduarda Calili ${ }^{9}$, ARRAIS, Maria Eduarda de Carvalho Sampaio ${ }^{10}$, ALVARES, Petra Paganucci Villarinho ${ }^{11}$, OLIVEIRA, Igor de Sousa ${ }^{12}$, RIBEIRO, Yana Sarah Fernandes Souza ${ }^{13}$, VASCONCELOS, Gilberto Loiola de ${ }^{14}$

ALMEIDA, Mariana Ferreira. Et al. Manejo da síndrome da veia cava superior. Revista Científica Multidisciplinar Núcleo do Conhecimento. Ano 06, Ed. 07, Vol. 11, pp. 134-149. Julho de 2021. ISSN: 2448-0959, Link de acesso: https://www.nucleodoconhecimento.com.br/saude/veia-cava-superior, $\quad$ DOI: 10.32749/nucleodoconhecimento.com.br/saude/veia-cava-superior

\section{RESUMO}

Objetivo: Identificar propostas terapêuticas benéficas e comprovadamente eficazes para o manejo do paciente com Síndrome da Veia Cava Superior (SVCS) mediante análise da literatura vigente. Métodos: Utilizando a base de dados Biblioteca Virtual

\footnotetext{
${ }^{1}$ Discente do curso de Medicina da Universidade Federal do Maranhão.

${ }^{2}$ Discente do curso de Medicina da Universidade de Ribeirão Preto.

${ }^{3}$ Discente do curso de Medicina do Centro Universitário Presidente Antônio Carlos.

${ }^{4}$ Discente do curso de Medicina da Universidade Federal do Maranhão.

${ }^{5}$ Discente do curso de Medicina da Universidade José do Rosário Vellano - UNIFENAS.

${ }^{6}$ Discente do curso de Medicina da Universidade Municipal de São Caetano do Sul.

${ }^{7}$ Discente do curso de Medicina da Universidade de São Caetano do Sul - USCS.

${ }^{8}$ Discente do curso de Medicina da Universidade Federal do Maranhão.

${ }^{9}$ Discente do curso de Medicina da Universidade do Sul de Santa Catarina.

${ }^{10}$ Discente do curso de Medicina do Centro Universitário UniFacid - UNIFACID.

${ }^{11}$ Discente do curso de Medicina da Universidade de São Caetano do Sul - USCS.

12 Discente do curso de Medicina da Universidade Federal de Campina Grande, Centro de Formação dos Professores, Cajazeiras - Paraíba.

${ }^{13}$ Discente do curso de Medicina do Centro Universitário INTA - UNINTA.

${ }^{14}$ Orientador. Médico residente em Clínica Médica, formado pela Universidade Federal do Ceará.
}

RC: 92216

Disponível em: https://www.nucleodoconhecimento.com.br/saude/veia-cava-superior 
de Saúde e os Descritores em Ciências da Saúde foram selecionados 9 artigos para análise. Resultados: A obstrução da Veia Cava Superior (VCS) está envolvida com variações anatômicas e diversas etiologias. Tendo em vista o caráter multifatorial da doença, os tratamentos empregados são diversificados e, dentre eles, os stents endovasculares são, majoritariamente, a opção de tratamento mais segura para aliviar os sintomas agudos em pacientes com SVCS. Além disso, o uso adjunto de trombólise sistêmica adjunto da colocação de stent via venografia pode manejar obstruções na VCS causadas pela aplicação de marca-passo. Observou-se, ainda, que o reparo endovascular percutâneo da veia cava é seguro e eficaz nos pacientes com SVCS. Conclusão: Dentre as abordagens terapêuticas aventadas, destacam-se a terapia endovascular com o uso de stents, além das terapias comumente utilizadas, a exemplo de radioterapia, uso de esteróides e quimioterapia.

Palavras-chave: Síndrome Da Veia Cava Superior, Obstrução da Veia Cava Superior, Trombose da Veia Cava Superior.

\section{INTRODUÇÃO}

A Síndrome da Veia Cava Superior (SVCS) é caracterizada por uma obstrução ou estenose da veia cava superior (VCS) que acarreta mau retorno venoso ou obstrução do fluxo venoso para a cabeça, parte superior, extremidades e tórax. A etiologia da SVCS abrange causas malignas, implicando o mediastino anterior ou medial, como câncer de pulmão, linfoma e câncer de mama, que representavam $90 \%$ dos casos que se apresentavam com SVCS. Além disso, pode-se apresentar com causas benignas, que de acordo com o crescente uso de cateteres venosos centrais de longa permanência e marcapasso cardíaco, a ocorrência de casos com causas não malignas está ampliando, representando mais de $40 \%$ dos casos de SVCS (CANALES et al., 2011) (GRAY, 2011).

Nessa perspectiva, são sinais e sintomas de mau retorno venoso o aparecimento de edema de facial e/ou cérvico facial e edema de membros superiores. Nesse sentido, podem-se citar também os sintomas como dispneia, tosse seca, dor de cabeça

RC: 92216

Disponível em: https://www.nucleodoconhecimento.com.br/saude/veia-cava-superior 
como sendo mais frequentes na apresentação da doença. Por outro lado, síncope, rouquidão, tontura e confusão são sintomas menos frequentes, mas que podem se manifestar.

Ademais, os pacientes com quadro de malignidade possuem maior predisposição para manifestar tosse, dispneia em repouso e dor no ombro, apresentando hiponatremia e perda de peso. Outrossim, a extensão e velocidade do estreitamento da VCS define o grau e a gravidade dos sintomas, sendo maiores os sintomas com o comprometimento da veia ázigos (GRAY, 2011) (RICE; RODRIGUEZ; LIGHT, 2006).

Nesse sentido, a SVCS reduz o retorno venoso proveniente da cabeça, pescoço e dos membros superiores e evidencia sintomas anteriormente citados e evidentes, a exemplo, principalmente, edema da face e/ou pescoço, dilatação e aspecto torto das veias e vênulas dessas regiões. Nesse contexto, evidencia-se a importância de reconhecer de forma precoce, bem como manejar o paciente de maneira satisfatória, uma vez que o prognóstico benéfico está diretamente correlacionado com o entendimento e a conduta terapêutica correta em curto prazo. Com isso, salienta-se a seguinte questão: "Quais as condutas terapêuticas de maior relevância atual diante da Síndrome da Veia Cava Superior, bem como as consequências significativas dos manejos propostos?".

Isso se evidencia, uma vez que em caso não diagnosticado, a SVCS pode levar a uma obstrução significativa das vias áreas e da circulação sanguínea e o paciente desenvolve o risco de ter uma parada cardíaca ou insuficiência respiratória. Por isso, deve-se observar sinais de alertas para tal situação, a exemplo edema laríngeo e edema cerebral correlacionado com cefaleia, necessitando de um tratamento imediato com diuréticos e dieta hipossódica, elevação da cabeceira e auxílio de oxigênio. Além disso, pode-se associar com 0 uso de glicocorticóides e, posteriormente, definir o tratamento definitivo.

RC: 92216

Disponível em: https://www.nucleodoconhecimento.com.br/saude/veia-cava-superior 
Desse modo, salienta-se que esse estudo tem como objetivo principal entender as formas mais benéficas de abordagem no manejo do paciente com a SVCS, permitindo um entendimento mais amplo das relações de benefícios existentes nessas condutas.

\section{METODOLOGIA}

Essa pesquisa se caracteriza como uma revisão bibliográfica do tipo integrativa de abordagem quanti-qualitativa, possuindo uma natureza aplicada com o objetivo de analisar o manejo da SVCS a partir de procedimentos bibliográficos. Dessa forma, essa revisão tem como finalidade identificar as condutas com maior eficácia e fornecer informações que permitam ao profissional da saúde ter uma abordagem mais ampla do paciente com a tal síndrome, contribuindo para que o paciente possa ter uma maior sobrevida.

Nessa perspectiva, durante o mês de abril de 2021 foi realizada uma pesquisa a partir da base de dados Biblioteca Virtual de Saúde (BVS) e sites aliados. Para isso, selecionaram-se descritores para formatar a fórmula de busca, por meio da base online Descritores em Ciências da Saúde (DeCS), sendo utilizados: "síndrome da veia cava superior", "obstrução da veia cava superior" e "trombose da veia cava superior", nos idiomas português, inglês e espanhol, bem como os seus sinônimos pertinentes e os operadores booleanos ("parênteses", "AND" e "OR"). Ademais, os filtros utilizados na pesquisa foram recorte temporal de cinco anos e idiomas, onde primeiramente foi utilizado o espanhol, seguido do inglês e por último o português. Desse modo, após a aplicação de todos os filtros, restaram 35 artigos na base de dados utilizada.

Por conseguinte, para a elegibilidade dos artigos resultantes na BVS foram utilizados os seguintes critérios: incluíram-se artigos que elucidassem pacientes, crianças ou adultos, que apresentassem SVCS e que abordassem os principais métodos terapêuticos para tal doença, inclusas no recorte temporal pré-determinado. Por outro lado, excluíram-se artigos que abordassem outros pontos além da terapêutica

RC: 92216

Disponível em: https://www.nucleodoconhecimento.com.br/saude/veia-cava-superior 
da síndrome, bem como artigos de opinião, metanálises, revisões sistemáticas, estudos duplicados e artigos que não estavam disponíveis na íntegra. Ressalta-se que a inclusão dos pacientes independia de etnia ou sexo.

Diante disso, a análise da busca foi realizada pelos pesquisadores, ISO e MFA, que avaliaram todos os títulos e resumos dos artigos, selecionando os estudos baseados nos critérios de elegibilidade previamente citados. Dessa forma, após a análise restaram 9 artigos da plataforma BVS que se adequaram aos critérios utilizados.

Nesse contexto, essa pesquisa não foi submetida ao Comitê de Ética, haja vista que é uma revisão bibliográfica e, portanto, não possui aplicação prática direta ou indireta com seres humanos ou animais.

Por fim, os dados obtidos e analisados na pesquisa serão apresentados nos resultados através da apresentação de um quadro organizado em ordem alfabética através do título do artigo, contendo os aspectos técnicos de cada um deles, sendo esses: título, ano e local de publicação, autoria e principais resultados.

\section{RESULTADOS}

\section{Quadro 01: Desfechos mais Relevantes dos Artigos Selecionados}

Título

Autores, Principais

ano de Resultados

publicação

e país

Artigo Trimodality Therapy for an Momozane

Advanced Thymic Carcinoma with et al., 2016, Both Aorta and Vena Cava Japão. Invasion.

Apesar de não existir (1)

RC: 92216

Disponível em: https://www.nucleodoconhecimento.com.br/saude/veia-cava-superior 


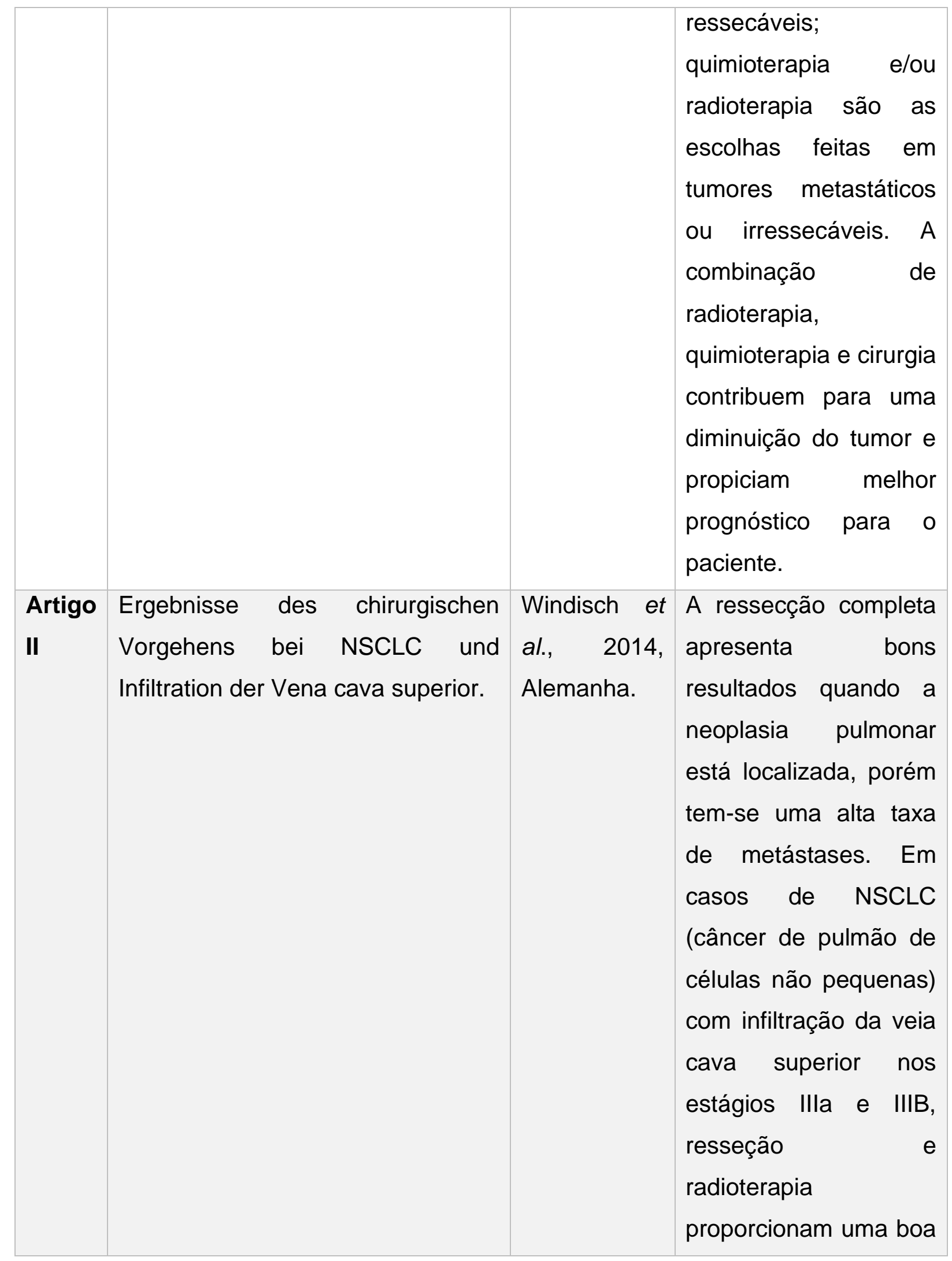

RC: 92216

Disponível em: https://www.nucleodoconhecimento.com.br/saude/veia-cava-superior 


\begin{tabular}{|c|c|c|c|}
\hline & & & $\begin{array}{ll}\text { alternativa } & \text { de } \\
\text { tratamento. } & \end{array}$ \\
\hline $\begin{array}{l}\text { Artigo } \\
\text { III }\end{array}$ & \begin{tabular}{lcr}
\multicolumn{3}{l}{ Superior vena cava syndrome after } \\
radiofrequency & sinus & node \\
modification & treated & with \\
thrombolysis & and & stent \\
implantation. & & \\
\end{tabular} & $\begin{array}{l}\text { Marciniak, } \\
\text { A.; } \\
\text { Gonsalves, } \\
\text { M.; } \\
\text { Gallagher, } \\
\text { M., 2015, } \\
\text { Inglaterra. }\end{array}$ & $\begin{array}{l}\text { O estudo evidenciou } \\
\text { que uma obstrução na } \\
\text { VCS (Veia Cava } \\
\text { Superior) causada pela } \\
\text { colocação de um } \\
\text { marca-passo pode ser } \\
\text { manejada com o uso } \\
\text { de trombólise } \\
\text { sistêmica } \\
\text { posteriormente, por } \\
\text { colocação de stent via } \\
\text { venografia. }\end{array}$ \\
\hline $\begin{array}{l}\text { Artigo } \\
\text { IV }\end{array}$ & $\begin{array}{l}\text { Successful treatment of central } \\
\text { venous catheter induced superior } \\
\text { vena cava syndrome with } \\
\text { ultrasound accelerated catheter- } \\
\text { directed thrombolysis. }\end{array}$ & $\begin{array}{l}\text { Dumantepe, } \\
\text { M.; Tarhan, } \\
\text { A.; Ozler, A., } \\
\text { 2013, } \\
\text { Turquia. }\end{array}$ & $\begin{array}{l}\text { Stents endovasculares } \\
\text { são, majoritariamente, } \\
\text { a opção de tratamento } \\
\text { mais segura para } \\
\text { aliviar os sintomas } \\
\text { agudos em pacientes } \\
\text { com SVCS. }\end{array}$ \\
\hline $\begin{array}{l}\text { Artigo } \\
\text { V }\end{array}$ & $\begin{array}{l}\text { Un syndrome cave supérieur } 12 \\
\text { ans après l'implantation d'un } \\
\text { pacemaker. }\end{array}$ & $\begin{array}{l}\text { Espitia et al., } \\
\text { 2012, } \\
\text { França. }\end{array}$ & $\begin{array}{l}\text { As investigações } \\
\text { diagnósticas em } \\
\text { pacientes suspeitos de } \\
\text { svcs devem ser feitas } \\
\text { por venografia em } \\
\text { situações onde a } \\
\text { flebotomia foi falha. }\end{array}$ \\
\hline $\begin{array}{l}\text { Artigo } \\
\text { VI }\end{array}$ & $\begin{array}{l}\text { A case of pacemaker related } \\
\text { superior vena cava syndrome after }\end{array}$ & $\begin{array}{l}\text { Sainathan, } \\
\text { S.; Andaz, }\end{array}$ & $\begin{array}{l}\text { O estudo evidencia } \\
\text { que os fios do marca- }\end{array}$ \\
\hline
\end{tabular}

RC: 92216

Disponível em: https://www.nucleodoconhecimento.com.br/saude/veia-cava-superior 


\begin{tabular}{|c|c|c|c|}
\hline & thoracic surgery. & $\begin{array}{l}\text { S., } 2012, \\
\text { Estados } \\
\text { Unidos. }\end{array}$ & $\begin{array}{l}\text { passo danificaram } \\
\text { cronicamente os } \\
\text { vasos, predispondo o } \\
\text { paciente a trombose } \\
\text { aguda nas veias } \\
\text { centrais. }\end{array}$ \\
\hline $\begin{array}{l}\text { Artigo } \\
\text { VII }\end{array}$ & $\begin{array}{l}\text { Neoplastic superior vena cava } \\
\text { obstruction: combined approach. }\end{array}$ & $\begin{array}{l}\text { Rose, } \quad \text { D.; } \\
\text { Santo, } \quad \text { C.; } \\
\text { Frati, } \quad \text { G., } \\
2011 \text {, Itália. }\end{array}$ & $\begin{array}{l}\text { A abordagem de } \\
\text { pacientes com SVCS } \\
\text { por neoplasias deve } \\
\text { ser múltipla e incluir } \\
\text { quimioterapia, } \\
\text { radioterapia e cirurgia } \\
\text { se o tumor for } \\
\text { ressecável. }\end{array}$ \\
\hline $\begin{array}{l}\text { Artigo } \\
\text { VIII }\end{array}$ & $\begin{array}{l}\text { Is stenting for superior vena cava } \\
\text { syndrome } \\
\text { supercalifragilisticexpialidocious? }\end{array}$ & $\begin{array}{l}\text { Gray, } \\
2011, \\
\text { Estados } \\
\text { Unidos. }\end{array}$ & $\begin{array}{l}\text { Demonstra-se } \\
\text { benefício no uso de } \\
\text { terapia trombolítica ou } \\
\text { angioplastia, bem } \\
\text { como na abordagem } \\
\text { cirúrgica de tumores } \\
\text { ressecáveis } \\
\text { causadores de SVCS. }\end{array}$ \\
\hline $\begin{array}{l}\text { Artigo } \\
\text { IX }\end{array}$ & $\begin{array}{l}\text { Single center experience with } \\
\text { percutaneous endovascular repair } \\
\text { of superior vena cava syndrome. }\end{array}$ & $\begin{array}{l}\text { Canales et } \\
\text { al., 2011, } \\
\text { Estados } \\
\text { Unidos. }\end{array}$ & $\begin{array}{l}\text { Reparo endovascular } \\
\text { percutâneo da veia } \\
\text { cava é seguro e eficaz } \\
\text { nos pacientes com } \\
\text { SVCS, com ou sem } \\
\text { uso de terapias } \\
\text { adjuvantes; não foram } \\
\text { evidenciadas mortes }\end{array}$ \\
\hline
\end{tabular}

RC: 92216

Disponível em: https://www.nucleodoconhecimento.com.br/saude/veia-cava-superior 


\begin{tabular}{|l|l|l|}
\hline & ou complicações \\
consecutivas à \\
abordagem.
\end{tabular}

Fonte: Autoria Própria, 2021.

Mediante a análise dos estudos selecionados, verificou-se que, pelas variações anatômicas envolvidas na obstrução da VCS, bem como pelas diversas etiologias associadas, ainda não há uma estratégia isolada e bem definida de manejo de pacientes portadores da SVCS (DUMANTEPE et al., 2013).

Dentre os principais desfechos observados, notou-se que a utilização de anticoagulação isolada em pacientes portadores de SVCS não desencadeou resultados significativos na maioria dos pacientes, apesar da demonstração de eficácia em casos brandos da doença. Neste mesmo estudo, Dumantepe et al. (2013) apontam que a terapia endovascular definitiva deve anteceder a anticoagulação vitalícia, uma vez que a primeira executa um papel fundamental na manutenção da circulação colateral e, portanto, reduz a ocorrência de reoclusão. Os autores salientam, ainda, o êxito decorrente da utilização de stents endovasculares, o que fica evidente pela redução aguda dos sintomas de pacientes que foram submetidos à aplicação precocemente, em até 5 dias do início dos sintomas.

No que concerne às limitações à realização da terapia endovascular com aplicação de stents, exemplificadas pelos pacientes com trombos de grande calibre, orienta-se a trombectomia ou a trombólise antes da inserção do stent. Neste sentido, observouse que a Trombólise Dirigida por Cateter Acelerada por Ultrassonografia (UACDT) pode ser utilizada como aliada, por promover elevação da penetração da droga trombolítica no trombo e, consequentemente, fragilizar a matriz de fibrina, dispensando a necessidade de terapêutica trombolítica intravenosa sistêmica, o que reduz consideravelmente o tempo de hospitalização e as complicações relacionadas a sangramentos, principalmente hematomas e hemorragias gastrointestinais. Portanto, a análise dos artigos selecionados para esta pesquisa permite atestar o

RC: 92216

Disponível em: https://www.nucleodoconhecimento.com.br/saude/veia-cava-superior 
êxito no tratamento da SVCS em pacientes sintomáticos que foram submetidos à UACDT e ao uso de varfarina em longo prazo. Logo, a angioplastia adjuvante e o implante de stent na porção da VCS estenótica residual são responsáveis por vantagens importantes na terapêutica da enfermidade (DUMANTEPE et al., 2013).

Em consonância ao estudo turco supracitado, Marciniak et al. (2016) também atestaram que a terapia anticoagulante, mesmo iniciada no momento do diagnóstico da SVCS, não revelou melhora significativa do quadro clínico, assim como promoveu aumento da carga trombótica dos pacientes estudados. Em contrapartida, fez-se necessária intervenção trombolítica sistêmica, a qual propiciou resolutividade trombótica quando associada à inserção de stents, o que resultou em melhora clínica após 12 horas.

Ademais, estudo realizado pela Escola de Medicina da Universidade da Carolina do Sul aponta a terapia trombolítica como uma alternativa de manejo em pacientes com sintomatologia aguda repentina desencadeada por obstrução da VCS. Os riscos associados a esta terapia podem ser minimizados mediante a utilização de cateteres de infusão isolada, os quais limitam a distribuição sistêmica e a biodisponibilidade errática da dose. Cabe ressaltar, ainda, os desfechos positivos relacionados à realização de angioplastia e de implante de stents, intervenções que demonstraram melhorar morbidade e mortalidade associadas à revascularização cirúrgica. Soma-se a isto a possibilidade de emprego de quimioterapia, radioterapia e outras terapêuticas alternativas combinadas em pacientes com SVCS maligna em uso de terapia endovascular (GRAY, 2011).

Neste cenário, verifica-se que, apesar da baixa incidência, os tumores cardíacos, majoritariamente benignos, podem configurar fator de predisposição ao desenvolvimento de SVCS. Pode-se citar com maior ênfase, neste sentido, a relação entre o angiossarcoma (tumor com incidência de $33 \%$ dentre os malignos) e a SVCS. Por localizar-se no átrio direito, o tumor pode resultar no bloqueio sanguíneo do lado direito, desencadeando a síndrome em foco. Evidenciou-se benefício, nos pacientes portadores da neoplasia, em realizar ressecção cirúrgica precedida por

RC: 92216

Disponível em: https://www.nucleodoconhecimento.com.br/saude/veia-cava-superior 
exames de imagens, tais como ressonância magnética e arteriografia com cateterismo coronário múltiplo, além de quimioterapia e radioterapia após ressecção, com o fito de melhorar sobrevida (ROSE et al., 2011).

No que se refere às entidades etiológicas relacionadas ao desenvolvimento da SVCS, vale salientar que a abordagem de acometimentos neoplásicos pode se dar por modalidade conservadora ou invasiva. Nesta conjuntura destaca-se o Carcinoma Tímico, enfermidade maligna que sujeita seus portadores a taxas de sobrevida de 30 a 75\% em 5 anos. Evidenciou-se, a partir da análise dos estudos selecionados, que o tratamento padrão preferível para tais neoplasias, quando ressecáveis, é a ressecção cirúrgica, em detrimento da operação de citorredução. No entanto, em tumores irressecáveis ou metastáticos preconiza-se a utilização de quimioterapia, radioterapia, ou a associação entre ambas. Cabe salientar, ainda, que a ressecção completa do tumor demonstrou ser fator prognóstico importante por possibilitar aumento de sobrevida dos pacientes acometidos, especialmente aqueles submetidos à quimiorradioterapia neoadjuvante (MOMOZANE et al., 2016).

No que tange às abordagens menos invasivas, estudo de Canales et al. (2012) analisou 14 pacientes portadores de SVCS submetidos ou não a terapias adjuvantes (implantes de stents, trombectomia ou trombólise), dentre os quais houve sucesso angiográfico em 93\%, melhora dos sintomas em 86\% e perviedade vascular após 12 meses em $82 \%$. Destaca-se, portanto, que a angioplastia vascular percutânea (PTS) associada a implante de stents endovasculares em pacientes com invasão de veia cava superior é segura e eficaz. Tal asserção é corroborada por trabalho de Sainathan e Andaz (2012), o qual aborda o desenvolvimento de SVCS relacionada ao uso de marca-passo em pós-operação torácica e atesta menor morbidade atrelada à terapia endovascular quando comparada à reconstrução cirúrgica aberta.

Por fim, estudo alemão que analisou retrospectivamente 22 indivíduos com neoplasia pulmonar não pequenas-células (NPNPC) submetidos à reconstrução ou à substituição de VCS constatou taxas de mortalidade de $7 \%$ em pacientes que realizaram pneumectomia e de $0 \%$ naqueles que sofreram lobectomia, assim como

RC: 92216

Disponível em: https://www.nucleodoconhecimento.com.br/saude/veia-cava-superior 
taxas de sobrevida em 5 anos de $21,4 \%$ e de $37,5 \%$ pós-pneumectomia ou lobectomia, respectivamente. Isto permite afirmar que a ressecção radical de VCS em pacientes portadores da SVCS comórbida à NPNPC possibilita significativo controle tumoral, a despeito da necessidade de utilização de quimioterapia adjuvante em uma quantidade considerável de pacientes (WINDISCH et al., 2015).

\section{DISCUSSÃO}

\section{MANEJO DO PACIENTE COM SVCS}

A SVCS, como já explorado anteriormente, dispõe de diversas etiologias possíveis que configuram um mau retorno venoso ou uma obstrução do fluxo venoso. Com isso, há várias possibilidades de intervenções terapêuticas a depender do gênero das suas respectivas causas da SVCS, o que implica em alternativas diversas no manejo do paciente acometido para muitos nichos etiológicos.

De acordo com os estudos evidenciados em resultados, os stents endovasculares, nos últimos anos, têm sido intitulados a alternativa terapêutica mais segura quanto ao alívio da sintomatologia aguda em pacientes com SVCS. Em paralelo com outras emergências obstrutivas, como as oncológicas, o manejo do paciente pode abranger o uso de stents intravasculares, além das terapias comumente utilizadas para reduzir a obstrução maligna, a exemplo da radioterapia, esteróides e quimioterapia.

A inclusão de stents, ainda em emergência oncológicas, produz o mesmo alívio sintomático dentro de um a dois dias, tornando-se proeminente nesse cenário, uma vez que, ocasionalmente, têm sido a única opção em tumores obstrutivos recorrentes (HIGDON et al, 2018). Outrossim, a colocação de stents endovasculares em pacientes com SVCS se mostrou majoritariamente bastante eficaz em conjunto com outras abordagens terapêuticas, quando após a colocação dos stents, a quimioterapia e a radioterapia eram aplicadas - acarretando pacientes com vida mais longeva. Tal longevidade se deve ao fato dessas terapias promoverem, de

RC: 92216

Disponível em: https://www.nucleodoconhecimento.com.br/saude/veia-cava-superior 
maneira ainda desconhecida, a prolongação da pervidade no fluxo sanguíneo (LANCIEGO et al, 2009).

Em um relato de caso realizado em 2015 na Inglaterra, a trombólise sistêmica demonstrou ser eficiente no manejo com pacientes que fazem uso de marca-passo, haja vista que geralmente esse aparelho e outros, como cateteres endovenosos, são capazes de induzir eventos trombóticos pela compressão vascular da VCS. A literatura atual disserta que em casos de pacientes acometidos da SVCS com grandes trombos devido à introdução de dispositivos, a trombólise se apresenta como meio para reduzir o tamanho da obstrução, bem como o risco de embolização iminente. Já em casos mais brandos com trombos menores, o tratamento se resume à retirada do aparelho endovascular e anticoagulação sistêmica para evitar a propagação do trombo (CUI et al, 2015).

A ressecção cirúrgica surge como uma das potenciais intervenções terapêuticas no manejo de pacientes que manifestam neoplasias malignas, como tumores do mediastino anterior ou medial e câncer de pulmão de células não pequenas (CPCNP). Em tumores cardíacos primários, quando malignos, a ressecção exibiu alívio de sintomas locais e permitiu a extração completa dos tecidos afetados, além de proporcionar o dobro da expectativa de vida em pacientes que realizam a ressecção em relação com aqueles que não a realizam, considerando, assim, a ressecção como tratamento de escolha. Quanto à ressecção em CPCNP, o estudo demonstrou que ela tende a ser útil em casos mais complexos, dado que diminuiu os índices de recorrência local na área de anastomose com redução de 92,9\% após lobectomia e de $90,9 \%$ após pneumonectomia. Ademais, o manejo no tratamento da CPCNP com a ressecção mostrou ser eficiente quando a quimioterapia atuava como neoadjuvante e a radioterapia como adjuvante, o que elevou a eficácia terapêutica nesses casos, pois essa associação propiciou efeitos positivos contra a elevada taxa de metástases. Para comprovar todas as evidências supracitadas, Roberts et al (1999) afirmou que, em causas malignas de SVCS, pacientes que passavam por cirurgia tinham uma taxa de sobrevivência de 5 anos de 13 a $31 \%$, enquanto

RC: 92216

Disponível em: https://www.nucleodoconhecimento.com.br/saude/veia-cava-superior 
aqueles que não passavam chegavam a apenas até $5 \%$, tal perspectiva sugeriu, então, que a ressecção apresentava ser uma opção viável para a SVCS.

\section{PROGNÓSTICO DO PACIENTE COM SVCS}

Como mencionado anteriormente, cabe ressaltar, que os stents endovasculares são uma das abordagens mais usadas, no que se refere proporcionar um tratamento mais adequado (DUMANTEPE, 2013). Perante ao exposto, evidenciando os resultados apresentados na revisão de literatura, nota-se que estudos expõem, que geralmente esse pacientes que aderem essa terapêutica, principalmente nos casos dos portadores da SVCS de origem benigna, possuem mais probabilidades de sucessos, uma vez que são raros os casos de complicações, ou até mesmo são menores as taxas de risco nos casos de reestenose. Dessa forma, configura-se como um tratamento paliativo, na tentativa de evitar procedimentos cirúrgicos futuros, consequentemente aumentando a expectativa de vida desses portadores, uma vez que, nota-se ao realizarem esse procedimento, em seguida apresentam alívio e melhora de sintomas agudo, contribuindo para o manejo e acompanhamento desses pacientes, (CARDOZO, 2006).

No que tange às pesquisas em relação da abordagem quimioterápicas e radioterápicas desses pacientes, é que essas intervenções facilitam a redução do tumor e permitem um melhor prognóstico (MOMOZANE, 2016). Somado a esse prisma, os estudos até hoje publicados apontam, que pacientes que apresentam carcinomas ou a presença de algum linfoma, adere-se uma terapêutica sistema com quimioterapia, uma vez que é muito eficiente no tratamento no casos mais graves, apesar de não ser um prognóstico favorável.

Diante disso, vale destacar também, que dependendo do quadro da doença, no que se refere ao seu estágio evolutivo e ao grau de obstrução, às vezes é necessário a ressecção do tumor. Nota-se que os indivíduos apresentam uma considerável resposta clínica, garantido bons resultados. Entretanto, impende salientar, que existem possibilidades de falhas podendo estar relacionados a presença de trombos

RC: 92216

Disponível em: https://www.nucleodoconhecimento.com.br/saude/veia-cava-superior 
na veia cava superior, sendo imprescindível uma melhor investigação da doença, a fim de garantir melhor tratamento (CIRINO, 2005). Ademais, em outra análise de evidências, os estudos apresentam, em relação às neoplasias pulmonares, a realização da ressecção completa (WINDISCH 2014).

Mediante ao exposto, é importante salientar que há as intervenções cirúrgicas, a exemplo da ressecção e do bypass. Nesses casos, os estudos publicados apontam que são maiores as incidências em pacientes que possuem complicações neoplásicas avançadas, o que contribui para maior incidência de risco mortalidade em decorrência dos tumores, uma vez que são considerados mau prognósticos para os pacientes. Dessa forma, torna-se necessário a realização da intervenção de ressecção desses indivíduos a fim de assegurar uma melhor longevidade (CIRINO, 2005).

\section{CONCLUSÃO}

A prevalência do manejo SVCS situa-se na terapia endovascular com o uso de stents. Notou-se que a utilização de anticoagulantes isolados em pacientes portadores de SVCS não desencadeou resultados significativos na maioria dos pacientes, apesar da demonstração de eficácia em casos brandos da doença.

O manejo do paciente pode abranger o uso de stents intravasculares, além das terapias comumente utilizadas, a exemplo da radioterapia, esteroides e quimioterapia. No tratamento para reduzir a obstrução maligna com a quimioterapia o estudo relatou a atuação como neoadjuvante e a radioterapia como adjuvante, o que elevou a eficácia terapêutica nesses casos, pois essa associação propiciou efeitos positivos contra a elevada taxa de metástases.

No que consiste às limitações à realização da terapia endovascular com aplicação de stents, exemplificadas pelos pacientes com trombos de grande calibre, orienta-se a trombectomia ou a trombólise antes da inserção do stent. Portanto, a análise dos artigos selecionados para esta pesquisa permite atestar o êxito no tratamento da

RC: 92216

Disponível em: https://www.nucleodoconhecimento.com.br/saude/veia-cava-superior 
SVCS em pacientes sintomáticos que foram submetidos à UACDT e ao uso de varfarina a longo prazo.

\section{REFERÊNCIAS}

CANALES, John F. et al. Single Center Experience with Percutaneous Endovascular Repair of Superior Vena Cava Syndrome. Catheterization and Cardiovascular Interventions, [s. I.], n. 77, p. 733 - 739, 2011. DOl https://doi.org/10.1002/ ccd.22871. Disponível em: https://www.researchgate.net/publication/ 51482261_Neoplastic_superior_vena_cava_obstruction_Combined_approach. Acesso em: 12 maio 2021.

CARDOZO, M. Tratamento endovascular da síndrome da veia cava superior: $\begin{array}{llllll}\text { relato de caso } & \text { da }\end{array}$ <https://www.scielo.br/j/jvb/a/dph7kwz3PRk4tCDqzbxqLtp/?lang=pt>. Acesso em: 4 junho 2021

CIRINO, L. Tratamento da síndrome da veia cava superior, 2005. <https://www.scielo.br/j/jbpneu/a/hp7SnnBcW5pxpT7M4fmghkt/?lang=pt>. Acesso em: 4 junho 2021.

CUI et al. Catheter-directed Thrombolysis in Acute Superior Vena Cava Syndrome Caused by Central Venous Catheters, 2015 <https://pubmed.ncbi.nlm.nih.gov/25827021/> . Acesso em: 28 maio 2021.

DUMANTEPE, Mert et al. Successful Treatment of Central Venous Catheter Induced Superior Vena Cava Syndrome With Ultrasound Accelerated Catheter-Directed Thrombolysis. Catheterization and Cardiovascular Interventions, [s. I.], ed. 269 273, 2013. DOI https://doi.org/10.1002/ccd.24855. Disponível em: https://scihub.scihubtw.tw/10.1002/ccd.24855. Acesso em: 12 maio 2021.

RC: 92216

Disponível em: https://www.nucleodoconhecimento.com.br/saude/veia-cava-superior 
ESPITIAA, O. et al. Un syndrome cave supérieur 12 ans après l'implantation d'un pacemaker: Superior vena cava syndrome induced by pacemaker probes, 12 years after introduction. Journal des Maladies Vasculaires, [s. I.], n. 38, p. 193 - 197, 2013. DOI https://doi.org/10.1016/j.jmv.2013.01.004. Disponível em: https://scihub.scihubtw.tw/10.1016/j.jmv.2013.01.004. Acesso em: 12 maio 2021.

GRAY, Bruce. Is Stenting for Superior Vena Cava Syndrome Supercalifragilisticexpialidocious?. Catheterization and Cardiovascular Interventions, [s. I.], ano 77, p. 740 - 741, 2011. DOI https://doi.org/10.1002/ ccd.23071. Disponível em: https://sci-hub.scihubtw.tw/10.1002/ccd.23071. Acesso em: 12 maio 2021

HIGDON, M. Oncologic Emergencies: Recognition and Initial Management. Am Fam Physician, 2018. <https://pubmed.ncbi.nlm.nih.gov/30215936/>. Acesso em: 27 maio 2021

LANCIEGO, C. Endovascular stenting as the first step in the overall management of

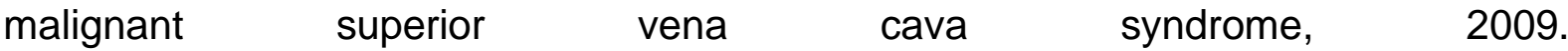
<https://pubmed.ncbi.nlm.nih.gov/25827021/>. Acesso em: 1 junho 2021.

MARCINIAK, A. et al. Superior vena cava syndrome after radiofrequency sinus node modification treated with thrombolysis and stent implantation. Departments of Cardiology and Radiology, St. George's Hospital, London, [s. I.], 2014. DOI https://doi.org/10.1093/europace/euu021. Disponível em: https://sci-hub.scihubtw.tw/ 10.1093/europace/euu021. Acesso em: 12 maio 2021.

MOMOZANE, Tohru et al. Trimodality Therapy for an Advanced Thymic Carcinoma With Both Aorta and Vena Cava Invasion. Robotic Cardiac Surgery: Mitral Valve Repair, Coronary Bypass \& More, [s. I.], v. 102, ed. 2, p. 139 - 141, 1 ago. 2016. DOI https://doi.org/10.1016/j.athoracsur.2016.01.014. Disponível em: https:// www.annalsthoracicsurgery.org/article/S0003-4975(16)00015-1/fulltext. Acesso em: 12 maio 2021

$\mathrm{RC}: 92216$

Disponível em: https://www.nucleodoconhecimento.com.br/saude/veia-cava-superior 
ROBERTS, J. Multimodality treatment of malignant superior vena caval syndrome. Chest, 1999 <https://pubmed.ncbi.nlm.nih.gov/10492298/>. Acesso em: 28 de maio 2021

ROSE, D. et al. Neoplastic superior vena cava obstruction: combined approach. European Review for Medical and Pharmacological Sciences, [s. I.], n. 15, p. 577 - 579, 2011. Disponível em: https:// www.researchgate.net/publication/ 51482261_Neoplastic_superior_vena_cava_obstruction_Combined_approach. Acesso em: 12 maio 2021.

SAINATHAN, SANDEEP; ANDAZ, SHAHRIYOUR. A Case of Pacemaker Related Superior Venacaval Syndrome after Thoracic Surgery. Department of Surgery, Bronx-Lebanon Hospital Center, Bronx, New York and Department of Cardiothoracic Surgery, South Nassau Communities Hospital, Oceanside, New York, [s. I.], 19 maio 2012. DOI 10.1111/j.1540-8159.2012.03520.x. Disponível em: https://sci-hub.scihubtw.tw/10.1111/j.1540-8159.2012.03520.x. Acesso em: 12 maio 2021.

WINDISCH, T. et al. Ergebnisse des chirurgischen Vorgehens bei NSCLC und Infiltration der Vena cava superior: Infiltration of the superior vena cava in NSCLC: Results of Surgical Intervention. Originalarbeit, [s. I.], p. 23 - 29, 6 nov. 2014. DOI http://dx.doi.org/10.1055/s-0034-1390806. Disponível em: https://sci-hub.st/10.1055/ s-0034-1390806. Acesso em: 12 maio 2021.

Enviado: Julho, 2021.

Aprovado: Julho, 2021.

RC: 92216

Disponível em: https://www.nucleodoconhecimento.com.br/saude/veia-cava-superior 